\title{
Neutrino-induced nucleosynthesis as a probe into the mechanism of supernovae
}

\section{Nadyozhin* and I. Panov}

A.I. Alikhanov Institute for Theoretical and Experimental Physics

E-mail: nadezhin@itep.ru, Igor.Panoveitep.ru

The neutrino nucleosynthesis yields depend on a number of factors. Apart from the onion-like presupernova chemical structure, they are sensitive to temporal and spectral properties of the neutrino "light curve" and to such hydrodynamic parameters as the supernova explosion energy and the delay in passing of the shock wave through successive presupernova chemical shells. Moreover, possible intrusions of other chemicals into the shells (e.g., hydrogen into the helium shell) can appreciably modify the final yields. We report the results of our systematic quantitative study of some of these effects with special attention to the supernova explosion energy and characteristic time of the neutrino flux.

International Symposium on Nuclear Astrophysics - Nuclei in the Cosmos - IX

25-30 June 2006

CERN

${ }^{*}$ Speaker. 


\section{Introduction}

The neutrino-induced nuclear processing in the envelopes of core-collapse supernovae is an important constituent of the theory of stellar nucleosynthesis. The resulting neutrino-dependent yields are sensitive to a number of parameters coupled both with the presupernova structure and the properties of not yet finally established details of the supernova mechanism. The most important ones are:

- The neutrino "light curve" and the neutrino energy and flavor spectra.

- The supernova total explosion energy $E_{\text {exp }}$ that determines the strength of the shock wave and time it takes to propagate through the presupernova envelope.

- The properties of the onion-like presupernova chemical structure, especially the distances of different chemical shells from stellar center.

The carbon-oxygen-neon shell and the helium one are good places to verify how the resulting yields depend on the above properties and thereby to obtain constraints on the supernova mechanism itself. Here we discuss the results we have obtained for the neutrino-induced nucleosynthesis in the helium shell.

\section{Method and Input Physics}

We have used two nuclear kinetics codes. The first one calculates the nuclear kinetics for the light nuclides from $\mathrm{D},{ }^{3} \mathrm{H},{ }^{3} \mathrm{He}, \ldots$ through ${ }^{24} \mathrm{Mg}$ (the $\mathrm{L}$ code). The second code $(\mathrm{H})$ controls the nuclear kinetic for the heavier nuclides up to $Z=60(\mathrm{Nd})$. The $\mathrm{L}$ code calculate the kinetics of about 160 thermonuclear reactions and beta-decays connecting 40 most important nuclides. The $\mathrm{H}$ code deals with about 1300 isotopes. Both the codes work consistently by exchanging with free neutrons and protons $[1,2]$.

The presupernova models were taken from [3]. The temporal behavior of the temperature, density, and radius of shocked material were described by analytical formulae from [4].

The following neutrino processes were taken into account.

Inelastic $\nu$ - $\alpha$ scattering:

${ }^{4} \mathrm{He}\left(v, v^{\prime} \mathrm{n}\right){ }^{3} \mathrm{He}, \quad{ }^{4} \mathrm{He}\left(v, v^{\prime} \mathrm{p}\right){ }^{3} \mathrm{H}$.

Inelastic $v$ scattering off ${ }^{12} \mathrm{C},{ }^{14} \mathrm{~N},{ }^{16} \mathrm{O}$, and ${ }^{20} \mathrm{Ne}$ :

${ }^{12} \mathrm{C}\left(v, v^{\prime 3} \mathrm{He}\right){ }^{9} \mathrm{Be}$,

${ }^{12} \mathrm{C}\left(v, v^{\prime} \mathrm{n}\right){ }^{11} \mathrm{C}, \quad{ }^{12} \mathrm{C}\left(v, v^{\prime} \mathrm{p}\right){ }^{11} \mathrm{~B}$,

${ }^{14} \mathrm{~N}\left(v, v^{\prime} \alpha\right){ }^{10} \mathrm{~B}$,

${ }^{16} \mathrm{O}\left(v, v^{\prime} \mathrm{n}\right){ }^{15} \mathrm{O}, \quad{ }^{16} \mathrm{O}\left(v, v^{\prime} \mathrm{p}\right){ }^{15} \mathrm{~N}$,

${ }^{20} \mathrm{Ne}\left(v, v^{\prime} \mathrm{n}\right){ }^{19} \mathrm{Ne}, \quad{ }^{20} \mathrm{Ne}\left(v, v^{\prime} \mathrm{p}\right){ }^{19} \mathrm{~F}$,

where $v$ stands for $\mu$ and $\tau$ neutrino and antineutrino.

Finally, the $\bar{v}_{\mathrm{e}}$ proton capture was included: $\mathrm{p}\left(\bar{v}_{\mathrm{e}}, \mathrm{e}^{+}\right) \mathrm{n}$. 
The total (bolometric) neutrino light curve was taken from [5]. The total neutrino energy radiated was assumed to be equally distributed among all the 6 neutrino and antineutrino species. The neutrino cross-sections, averaged over the Fermi-Dirac spectra with the zero chemical potential and temperature of $8 \mathrm{MeV}$ for $\mu, \tau$ neutrino and antineutrino and $4 \mathrm{MeV}$ for the electron neutrino and antineutrino, were taken from $[6,7,8]$.

\section{Results}

Figure 1 shows how the production factors (PF) change with the explosion energy $E_{\text {exp }}$. The $\mathrm{PF}$ for ${ }^{7} \mathrm{Li},{ }^{11} \mathrm{~B},{ }^{15} \mathrm{~N}$, and ${ }^{19} \mathrm{~F}$ include the contribution from beta-decays of their radioactive counterparts ${ }^{7} \mathrm{Be},{ }^{11} \mathrm{C},{ }^{15} \mathrm{O}$, and ${ }^{19} \mathrm{Ne}$, respectively. Although the PF dependence on $E_{\text {exp }}$ is noticeable, it is not crucial.

The ${ }^{11} \mathrm{~B}$ turns out to be always overproduced relative to ${ }^{7} \mathrm{Li}$. The reason is not yet clear and deserves further investigation in the framework of constraints on presupernova models and supernova mechanism.

The $\mathrm{PF}$ for ${ }^{9} \mathrm{Be}$ is small $(\sim 0.1)$. However, there is a hope to get $\mathrm{PF}\left({ }^{9} \mathrm{Be}\right)>1$ since the crosssection of the reaction ${ }^{12} \mathrm{C}\left(v, v^{13} \mathrm{He}\right){ }^{9} \mathrm{Be}$ is still poorly known and some, otherwise unimportant properties, of the presupernova structure may be helpful to resolve the problem. Moreover, the neutrino-induced production of ${ }^{9} \mathrm{Be}$ depends strongly on the duration of the neutrino "light" curve which in our calculations has a characteristic time of $\sim 20$ s [5] supported by the observations of SN 1987A. If one used the exponential approximation with the characteristic time $3 \mathrm{~s}$ the $\mathrm{PF}\left({ }^{9} \mathrm{Be}\right)$ would be at least by a factor of 10 lower. This occurs because there is not enough time in this case for the neutrino flux to restore ${ }^{9} \mathrm{Be}$ burned in the shock wave.

Figure 2 and 3 show the chemical structure of He-shells for $15 M_{\odot}$ and $75 M_{\odot}$ presupernova of very low metallicity: $Z=0.0001 Z_{\odot}$. Of special interest are the sub-shells with a rather small admixture of elements heavier than ${ }^{4} \mathrm{He}$ which used to absorb free neutrons and thereby prevent to build heavy elements from the "Fe" seeds. In terms of the mass coordinate $m$, the sub-shells are $m=(3.8-4.1) M_{\odot}$ and $m=(32.8-33.7) M_{\odot}$ for a $15 M_{\odot}$ and $75 M_{\odot}$ presupernova, respectively.

Figures 4, 5 show the results of our calculation of the neutrino nucleosynthesis in the sub-shell of a $15 M_{\odot}$ presupernova when all the species (but not the "Fe" seeds!) with the abundances below $\log X=-6$ are neglected (these abundances are not seen in Fig. 4). The initial abundance of the "Fe" seeds is $X_{\mathrm{Fe}}=3.15 \times 10^{-8}$. Thus in the metal-poor presupernovae, there exists a rather good site to obtain a kind of a "weak" $r$-process up to $A \approx 130$. Figure 5 demonstrates that the overall trend in the distribution of elemental abundances obtained in our calculations (a serrated solid line) is in good agreement with the solar and metal-poor star data.

If in the sub-shell of a $15 M_{\odot}$ presupernova the abundances of ${ }^{12} \mathrm{C},{ }^{16} \mathrm{O}$, and ${ }^{20} \mathrm{Ne}\left(X_{\mathrm{C} 12}=\right.$ $5.5 \times 10^{-8}, X_{\mathrm{O} 16}=1.2 \times 10^{-8}, X_{\mathrm{Ne} 20}=2.4 \times 10^{-7}$ ), dropped out in the previous calculation, are retained, then the neutrino-induced r-process extends to $A \approx 80$ only (Fig. 6 , solid line). The same story happens with a $75 M_{\odot}$ presupernova. A bulk of neutrons produced by the $v$-He spallation proves to be captured by a relatively abundant ${ }^{12} \mathrm{C}$ (Fig. 3) and only about one neutron being left for a "Fe" seed. However, for the ${ }^{12} \mathrm{C}$ abundance decreased by a factor of $\sim 10^{4}$ (in this case it 
becomes comparable with the abundance of ${ }^{16} \mathrm{O}$ ) we obtained a limited r-process shown in Fig. 6 by a dashed line.

\section{Conclusion}

The goal of this contribution is to draw the attention of the scientific community to the fact that some details of the presupernova models and of the mechanism of core-collapse supernovae can be probed by modelling the neutrino induced nucleosynthesis.

Three issues seem to be the most challenging for the helium shell:

- The synthesis of ${ }^{9} \mathrm{Be}$ that strongly depends on the total duration of the neutrino "light" curve.

- An overproduction of ${ }^{11} \mathrm{~B}$ relative to ${ }^{7} \mathrm{Li}$.

- The possibility of the neutrino-driven weak r-process in the poor-metal supernova models that depends on the admixture of such neutron poisons as ${ }^{12} \mathrm{C}$ and ${ }^{14} \mathrm{~N}$. Their concentrations might be sensitive to details of description of mixing in presupernova models (convective and semi-convective diffusion).

Acknowledgement. We thank the NIC-9 Organizing Committee for the invitation to attend the Conference. The work was supported by the grants: RFBR No. 04-02-16793 and SNSF No. IB7320-110996.

\section{References}

[1] D.K. Nadyozhin, I.V. Panov, and S.I. Blinnikov, The neutrino-induced neutron source in helium shell and r-process nucleosynthesis, Astron. Astrophys. 335, 207 (1998).

[2] D.K. Nadyozhin and I.V. Panov, A two-code iterative method to calculate the light and heavy element synthesis, Nuclear Phys., A688, 590c (2001).

[3] A. Heger and S.E. Woosley Grids of Stellar Evolution Models. Presupernova Structure Data Files., http://www.ucolick.org/ alex/stellarevolution/data.shtml

[4] D.K. Nadyozhin and A.Yu. Deputovich, An analytical approximation of post-shock conditions in type II supernova shells, Astron. Astrophys. 386, 711 (2002).

[5] D.K. Nadyozhin, The neutrino radiation for a hot neutron star formation and the envelope outburst problem, Astroph. Sp. Sci., 53, 131 (1978).

[6] A. Heger, E. Kolbe, W.C. Haxton et al. Neutrino Nucleosynthesis, Physics Letters, B606, 258 (2005); astro-ph/0307546 v1 $31 \mathrm{Jul} 2003$.

[7] S.E. Woosley, D.H. Hartmann, R.D. Hoffman, and W.C. Haxton, The nu-process, Astrophys. J., 356, 272 (1990).

[8] G.V. Domogatskij and S.V. Imshennik, Production of the ${ }^{9}$ Be isotope induced by neutrinos generated through gravitational stellar collapse, Sov. Astron. Lett., 8, 190, (1982).

[9] J.J. Cowan, C. Sneden, S. Burles et al., The chemical composition and age of the metal-poor halo star $B D+17^{\circ} 3248$, Astrophys. J., 572, 861 (2002). 


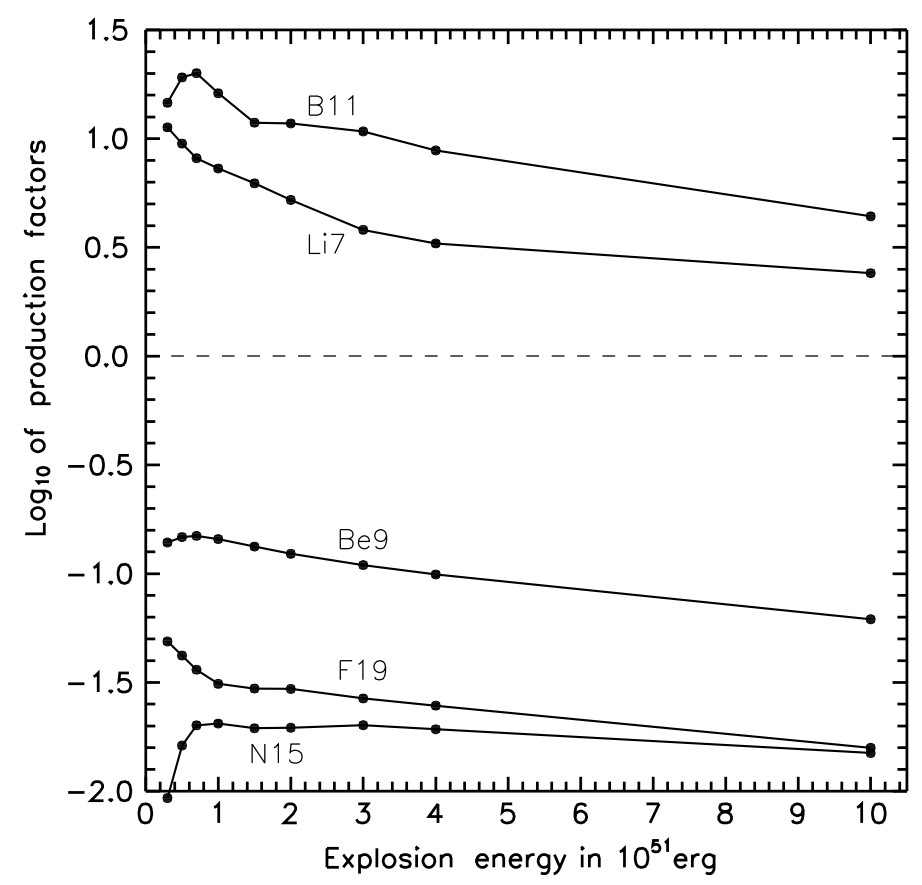

Figure 1: Production factors versus explosion energy in the helium shell of a $15 M_{\odot}$ supernova (solar metallicity).

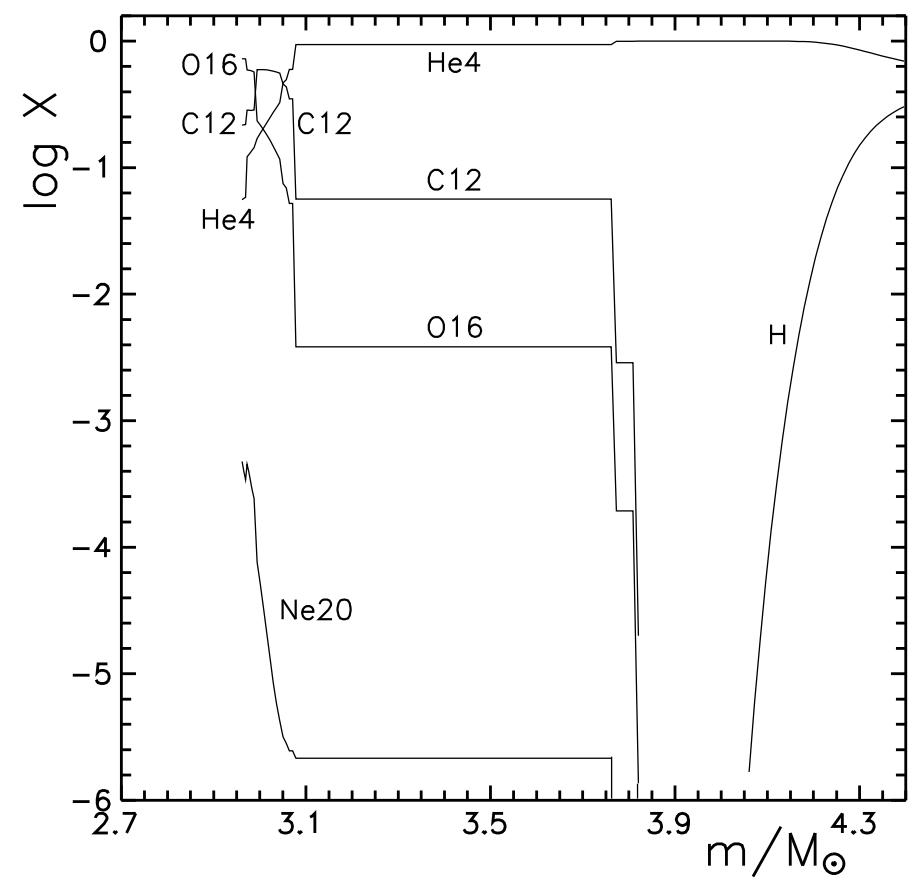

Figure 2: The composition of He-shell for a $15 M_{\odot}$ presupernova $\left(0.0001 Z_{\odot}\right)$ [3]. 


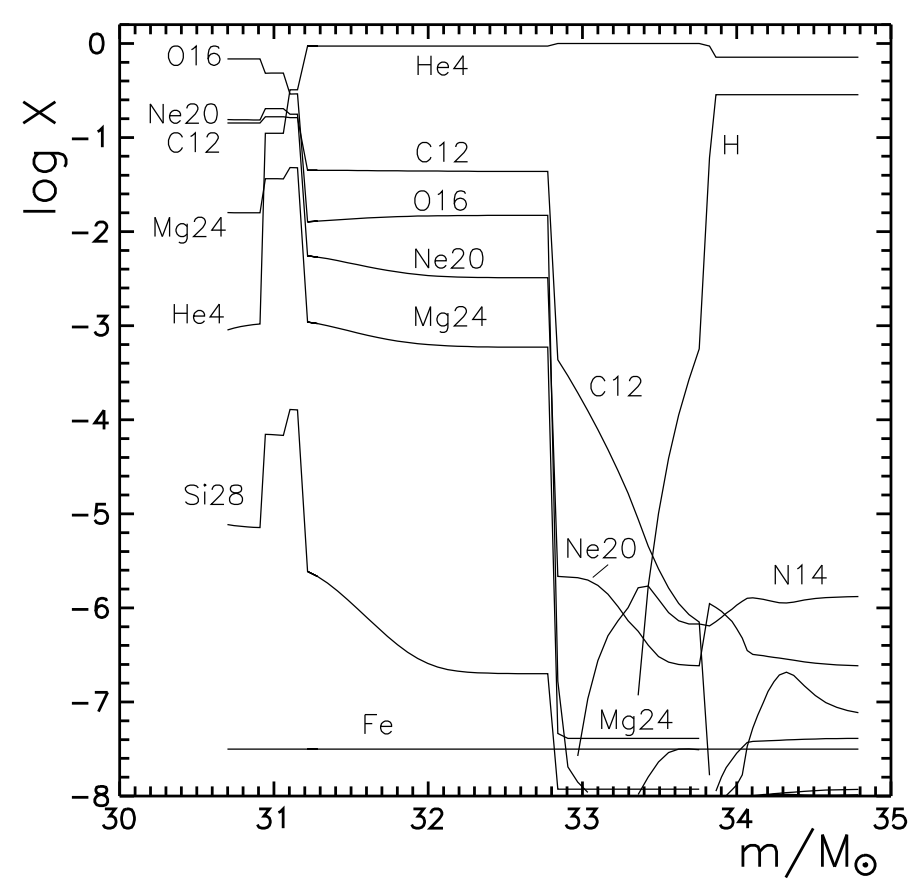

Figure 3: The composition of He-shell for a $75 M_{\odot}$ presupernova $\left(0.0001 Z_{\odot}\right)$ [3].

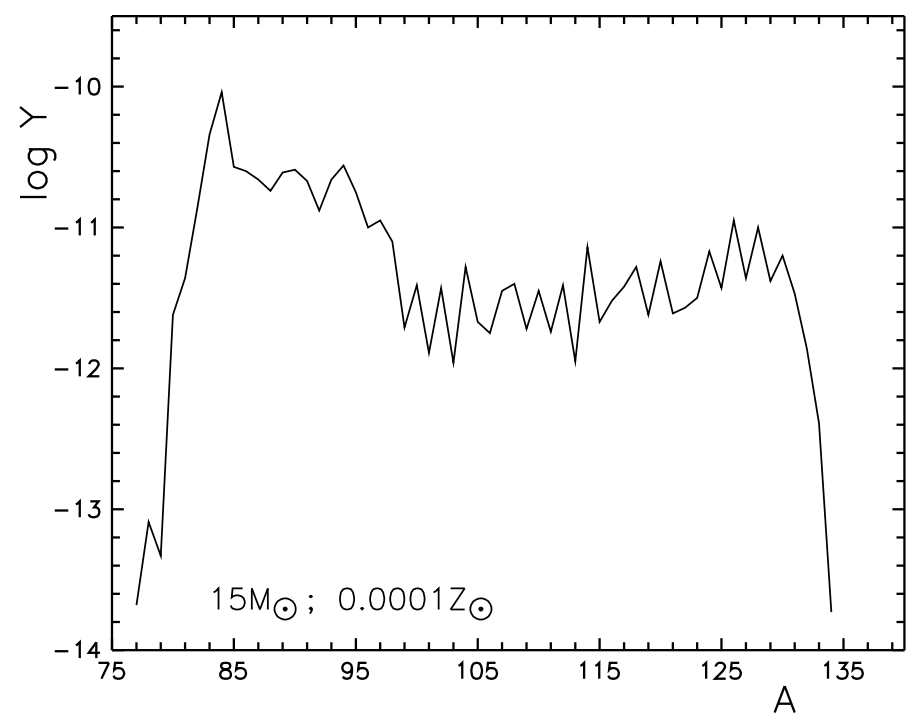

Figure 4: Heavy nuclides yields versus mass number $\mathrm{A}$ for a $15 M_{\odot}$ supernova $\left(0.0001 Z_{\odot}, E_{\exp }=\right.$ $1 \times 10^{51} \mathrm{erg}$ ). There were captured about 50 neutrons per "Fe" seed. 


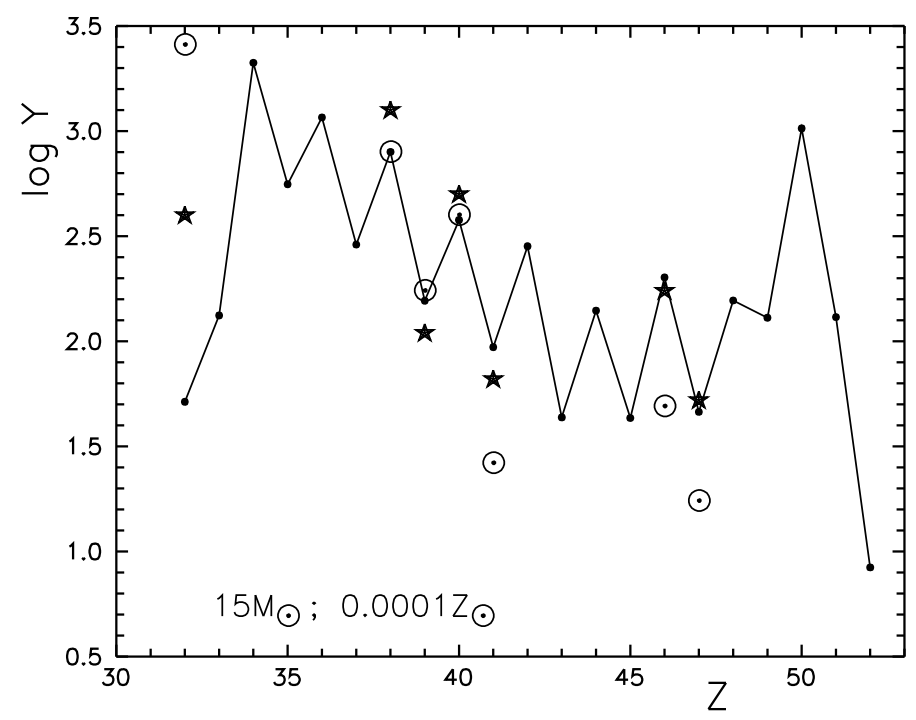

Figure 5: Heavy nuclide yields versus atomic number $\mathrm{Z}$ for a $15 M_{\odot}$ supernova $\left(0.0001 Z_{\odot}, E_{\exp }=\right.$ $1 \times 10^{51} \mathrm{erg}$ ). Black stars stand for a metal-poor star BD $+17^{\circ} 3248$ [9], also the solar abundances for some nuclides are shown. All the data are arbitrarily shifted along the vertical axis.

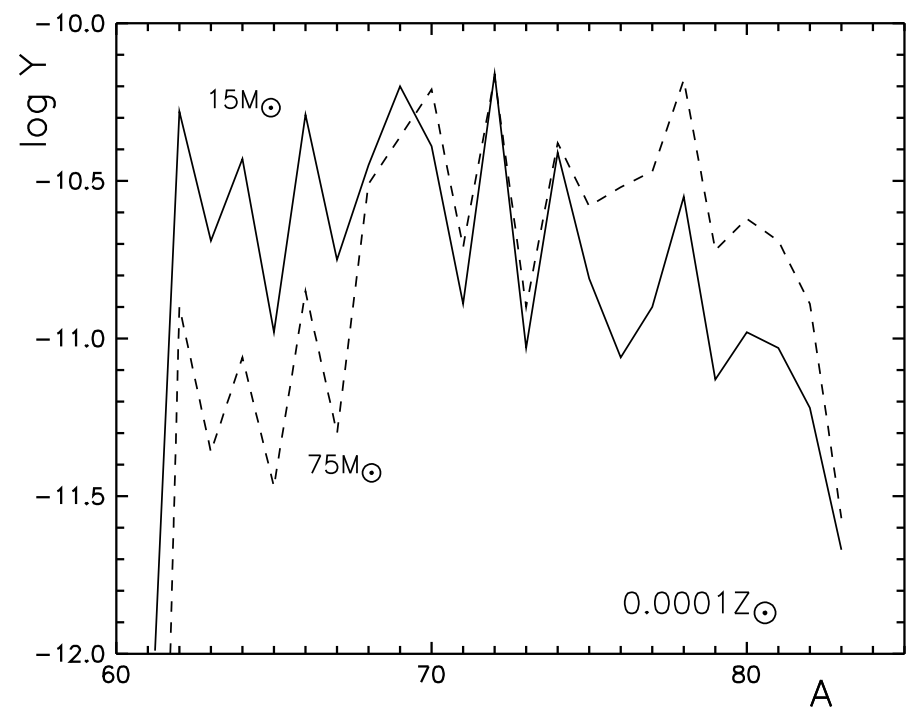

Figure 6: Heavy nuclide yields versus mass number $\mathrm{A}$ for a $15 M_{\odot}$ supernova $\left(0.0001 Z_{\odot}, E_{\exp }=\right.$ $\left.1 \times 10^{51} \mathrm{erg}\right)$ and a $75 M_{\odot}$ supernova $\left(0.0001 Z_{\odot}, E_{\exp }=1 \times 10^{52} \mathrm{erg}\right)$. About 14 and 17 neutrons were captured per "Fe" seed, respectively. 